\title{
On generalized fuzzy mean code word lengths
}

\author{
Dhara Singh Hooda, Arunodaya Raj Mishra, Divya Jain
}

Department of Mathematics, Jaypee University of Engineering and Technology, Guna, Madhya Pradesh, India

\section{Email address:}

ds_hooda@rediffmail.com (D. S. Hooda), arunodaya87@outlook.com (A. R. Mishra), agrawal_divya2002@yahoo.co.in (D. Jain)

\section{To cite this article:}

Dhara Singh Hooda, Arunodaya Raj Mishra, Divya Jain. On Generalized Fuzzy Mean Code Word Lengths. American Journal of Applied Mathematics. Vol. 2, No. 4, 2014, pp. 127-134. doi: 10.11648/j.ajam.20140204.13

\begin{abstract}
In present communication, a generalized fuzzy mean code word length of degree $\beta$ has been defined and its bounds in the term of generalized fuzzy information measure have been studied. Further we have defined the fuzzy mean code word length of type $(\alpha, \beta)$ and its bounds have also been studied. Monotonic behavior of these fuzzy mean code word lengths have been illustrated graphically by taking some empirical data.
\end{abstract}

Keywords: Entropy, Fuzzy Entropy, Codeword Length, Decipherable Code, Crisp Set, Hölder's Inequality

\section{Introduction}

Let $X$ be a discrete random variable with probability distribution $\quad P=\left\{\left(p_{1}, p_{2}, \ldots \ldots, p_{n}\right): p_{i} \geq 0 ; \sum_{i=1}^{n} p_{i}=1\right\} \quad$ in $\quad$ an experiment. [5] gave a mathematical formulation to measure uncertainty of the randomness in a probability distribution and information contained in an experiment as

$$
H(P)=-\sum_{i=1}^{n} p_{i} \log p_{i}
$$

which is called Shannon's entropy.

In many applications of the uncertainty function, the main problem generated by researchers is that of efficient coding of message to be sent over a noiseless channel, and to maximize the number of messages that can be sent over the channel in a given time. Let us consider that the messages to be transmitted are generated by a random variable $X=\left\{x_{1}, x_{2}, \ldots \ldots, x_{n}\right\}$ with the probability distribution $P=\left\{\left(p_{1}, p_{2}, \ldots \ldots, p_{n}\right): p_{i} \geq 0 ; \sum_{i=1}^{n} p_{i}=1\right\}$. Each $x_{i}$ is called source symbol or alphabet and is represented by a finite sequence of symbols select from the set $A=\left\{a_{1}, a_{2}, \ldots, a_{D}\right\}$. The set $A$ is known as code alphabet or set of code characters and the sequence assigned to each $x_{i} ; i=1,2, \ldots, n$ is called code word. The number of code character used for a code word is called code word length. Let $n_{i}$ be the code word length of $x_{i}$, then mean code word length is given

$$
L=\sum_{i=1}^{n} p_{i} n_{i}
$$

where $p_{i}$ is the probability of occurrence of $x_{i}$ satisfying Kraft's inequality

$$
\sum_{i=1}^{n} D^{-n_{i}} \leq 1
$$

where $D$ is the size of code alphabet.

In evaluating long run efficiency of communications, we choose codes to minimize average code word length (2).

For uniquely decipherable codes, [5] noiseless coding theorem states that

$$
\frac{H(P)}{\log D} \leq L \leq \frac{H(P)}{\log D}+1,
$$

which determines the lower and upper bounds on $L$ in terms of [5] entropy.

To prove noiseless coding theorem, [11] inequality plays an important role and is uniquely determined by the condition for uniquely decipherability. To tackle such situations, instead of taking the probability, the idea of fuzziness can be explored.

[10] Introduced the concept of fuzzy set in which imprecise knowledge can be used to define an event. Because of their capability to model non-statistical imprecision fuzzy set plays an important role in many systems A fuzzy set $A$ is a subset of $\mathrm{X}$ and is defined as 


$$
A=\left\{\left(x_{i}, \mu_{A}\left(x_{i}\right)\right): \mu_{A}\left(x_{i}\right) \in[0,1] ; \forall x_{i} \in X\right\},
$$

where $\mu_{A}\left(x_{i}\right)$ represents the degree of membership and is defined as

$$
\mu_{A}\left(x_{i}\right)=\left\{\begin{array}{l}
0, \text { if } x_{i} \notin A \text { and thereis noambiguity } \\
1, \text { if } x_{i} \in A \text { and thereis noambiguity } \\
0.5, \text { thereismaximumambiguity whether } x_{i} \notin A \text { or } x_{i} \in A
\end{array}\right.
$$

The idea of measuring fuzzy uncertainty without reference to probabilities began in 1972 with the work of [1] who defined the entropy of $A \in P_{n}(X)$ using Shannon's entropy as

$$
H(A)=-\sum_{i=1}^{n}\left[\mu_{A}\left(x_{i}\right) \log \mu_{A}\left(x_{i}\right)+\left(1-\mu_{A}\left(x_{i}\right)\right) \log \left(1-\mu_{A}\left(x_{i}\right)\right)\right]
$$

This is called fuzzy information measure. The fuzzy information measure has found wide applications to Engineering, Fuzzy traffic control, Fuzzy aircraft control, Computer sciences, management and Decision making, etc. and those have already been studied by various authors. [6] Introduced a new measure of fuzzy divergence explaining its application to clustering problems and to an object extraction problem.

In present paper, we define fuzzy mean code word lengths in section 2 . In section 3 , we also study the bounds of the generalized fuzzy mean code word length of degree $\beta$ in terms of fuzzy information measure and we study the bounds of the new fuzzy mean code word length of type $(\alpha, \beta)$ in terms of fuzzy information measure in section 4 . We discuss the monotonic behavior of generalized fuzzy mean code word lengths in section 5 .

\section{Fuzzy Mean Code Word Lengths}

[12] defined fuzzy mean code word length as given below:

$$
\bar{L}=\log \left[D^{n_{i} \mu_{A}\left(x_{i}\right)}\left(1-\frac{D^{-n_{i}}}{\sum_{j=1}^{n} D^{-n_{j}}}\right)^{-\left(1-\mu_{A}\left(x_{i}\right)\right)}\right]
$$
(5).

They studied the lower and upper bounds of $\bar{L}$ in term of Further based on [2], they generalized (6) as given below:

$$
\bar{L} \alpha=\sum_{i=1}^{n} \log \left[\frac{\alpha-\left\{\mu_{A}^{\alpha}\left(x_{i}\right)+\left(1-\mu_{A}\left(x_{i}\right)\right)^{\alpha}\right\}_{D}{ }^{-n_{i} \alpha}}{\alpha-1}\right] ; \alpha>0, \alpha \neq 1,
$$

which was called fuzzy mean code word length of order $\alpha$. Its lower and upper bounds were obtained in term of the following fuzzy information measure characterized by [6]:

$$
H_{\alpha}(A)=\frac{1}{1-\alpha} \sum_{i=1}^{n} \log \left[\mu_{A}^{\alpha}\left(x_{i}\right)+\left(1-\mu_{A}\left(x_{i}\right)\right)=[; \alpha>, \alpha \neq 1\right.
$$

[12] also defined fuzzy mean code word length of degree $\beta$ as given below:

$$
\bar{L}^{\beta}=\frac{1}{1-\beta} \sum_{i=1}^{n}\left[\left\{\mu_{A}^{\beta}\left(x_{i}\right)+\left(1-\mu_{A}\left(x_{i}\right)\right)^{\beta}\right\} D^{n_{i}\left(\frac{1-\beta}{\beta}\right)}-1\right] ; \beta>0, \beta \neq 1,
$$

which was called fuzzy mean code word length of degree $\beta$ and studied its lower and upper bounds in term of fuzzy information defined by [9] and is given as

$$
{ }_{H}^{\beta}(A)=\frac{1}{1-\beta} \sum_{i=1}^{n}\left[\mu_{A}^{\beta}\left(x_{i}\right)+\left(1-\mu_{A}\left(x_{i}\right)\right)^{\beta}-1\right] ; \beta>0, \beta \neq 1 .
$$

Corresponding to [3], [8] proposed and studied the measure of fuzzy entropy as given below:

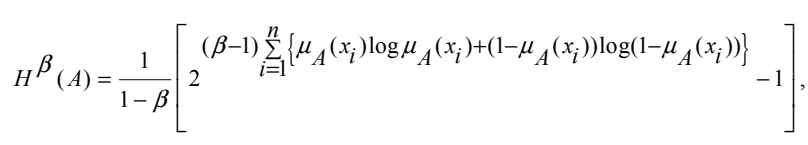

where

$$
\beta>0, \beta \neq 1
$$

Corresponding to [4], [7] studied generalized sub additive fuzzy information of type $(\alpha, \beta)$ given by

$$
H_{\alpha}^{\beta}(A)=\frac{1}{2^{1-\alpha}-2^{1-\beta}} \sum_{i=1}^{n}\left[\mu_{A}^{\alpha}\left(x_{i}\right)+\left(1-\mu_{A}\left(x_{i}\right)\right)^{\alpha}-\mu_{A}^{\beta}\left(x_{i}\right)-\left(1-\mu_{A}\left(x_{i}\right)\right)^{\beta}\right],
$$

where

$$
0<\alpha<1, \beta \geq 1 \text { or } 0<\beta<1, \alpha \geq 1 \text {. }
$$

\section{Bounds of a Generalized Fuzzy Mean Code Word Length of Degree $\beta$}

It may be noted that (6) can be generalized in various ways; however, we consider the following generalization:

$$
\frac{-\beta}{L}=\frac{1}{1-\beta}\left[2 \sum_{i=1}^{n}\left[\begin{array}{l}
\sum_{i=1}^{n} \log \\
\left.n_{i} \mu_{A}\left(x_{i}\right)\left(1-\frac{D^{-n_{i}}}{\sum_{j=1}^{n} D^{-n_{j}}}\right)^{-\left(1-\mu_{A}\left(x_{i}\right)\right)}\right\}
\end{array}\right]\right.
$$

where $\beta>0, \beta \neq 1$

and study its bounds in terms of (11).

Theorem 1. For all uniquely decipherable codes, noiseless coding theorem states:

$$
{ }_{H}^{\beta}(A) \leq \bar{L}^{\beta} \leq H^{\beta}(A) 2^{(1-\beta)}+(1-\beta)^{-1}\left(2^{(1-\beta)}-1\right),
$$

with equality if and only if 


$$
\mu_{A}\left(x_{i}\right)=\frac{D^{-n_{i}}}{\sum_{j=1}^{n} D^{-n_{j}}},
$$

where $H^{\beta}(A)$ is given by (11).

Proof. [7] have given the following expression for directed divergence:

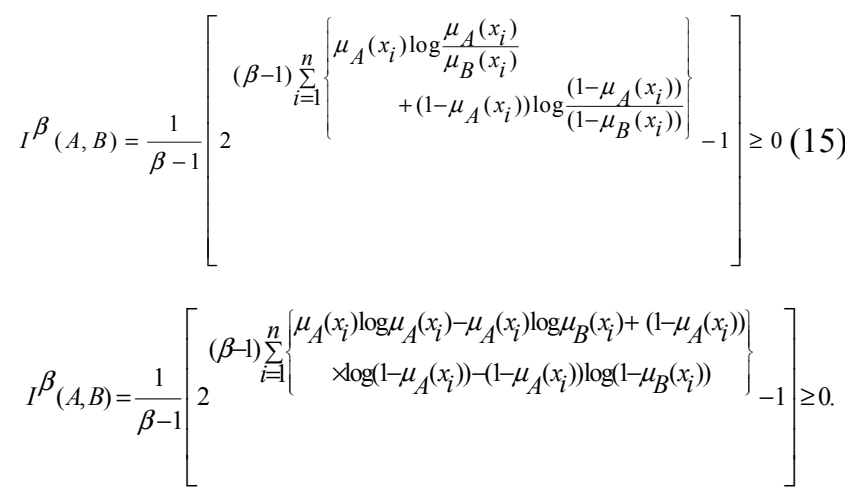

Taking $\mu_{B}\left(x_{i}\right)=\frac{D^{-n_{i}}}{\sum_{j=1}^{n} D^{-n_{j}}}, 1 \leq i \leq n$,

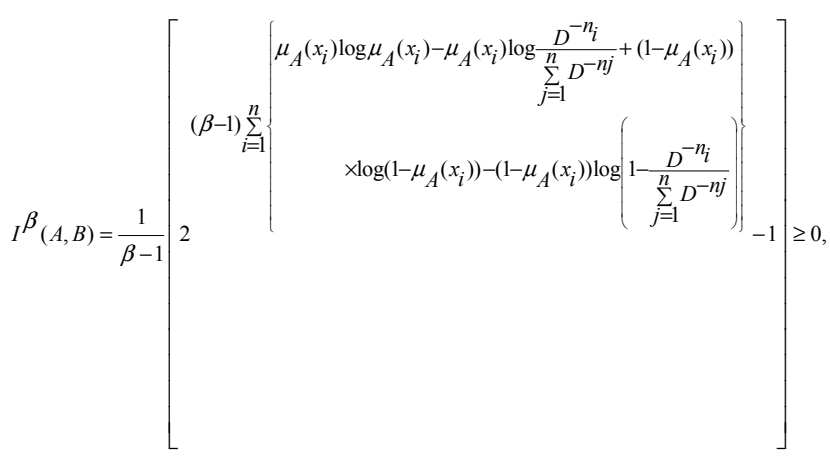

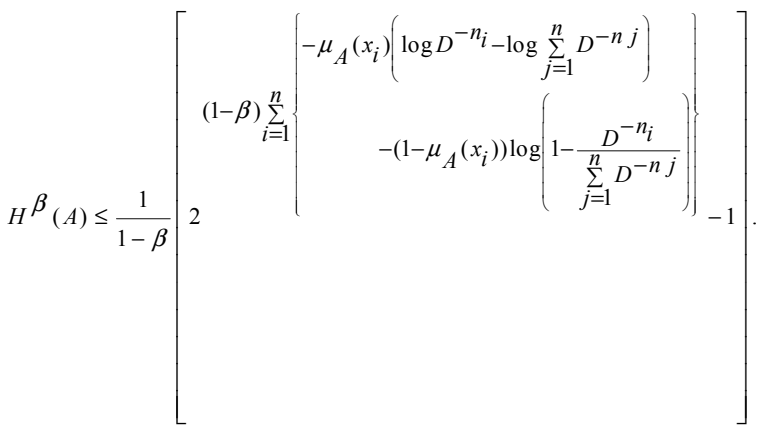

(16)

Using Kraft's inequality, that is $\sum_{j=1}^{n} D^{-n_{j}} \leq 1$, we get

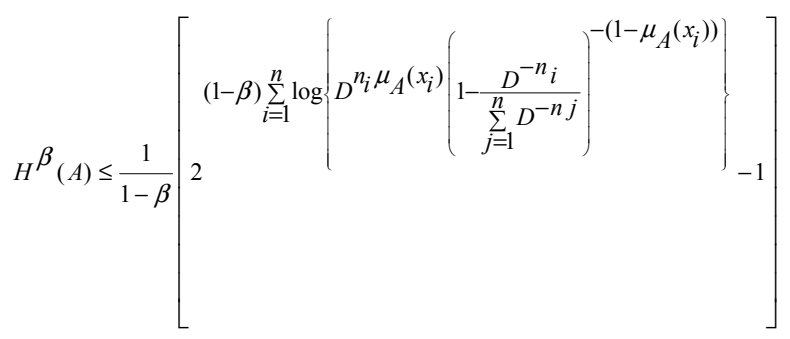

$$
{ }_{H}^{\beta}(A) \leq \bar{L} \beta
$$

For uniquely decipherable code, [5] noiseless coding theorem for fuzzy information measure as

$$
H(A) \leq \bar{L} \leq H(A)+1 .
$$

Then we have

$$
\bar{L} \leq H(A)+1
$$

It implies

$$
\begin{aligned}
& \sum_{i=1}^{n} \log \left[D^{n_{i} \mu_{A}\left(x_{i}\right)}\left\{1-\frac{D^{-n_{i}}}{\sum_{j=1}^{n} D^{-n_{j}}}\right\}^{-\left(1-\mu_{A}\left(x_{i}\right)\right)}\right] \\
& \leq-\sum_{i=1}^{n}\left[\mu_{A}\left(x_{i}\right) \log \mu_{A}\left(x_{i}\right)+\left(1-\mu_{A}\left(x_{i}\right)\right) \log \left(1-\mu_{A}\left(x_{i}\right)\right)\right]+1 \\
& (1-\beta) \sum_{i=1}^{n} \log \left[D^{n_{i} \mu_{A}\left(x_{i}\right)}\left\{1-\frac{D^{-n_{i}}}{\sum_{j=1}^{n} D^{-n_{j}}}\right\}^{-\left(1-\mu_{A}\left(x_{i}\right)\right)}\right] \\
& \leq(\beta-1) \sum_{i=1}^{n}\left[\mu_{A}\left(x_{i}\right) \log \mu_{A}\left(x_{i}\right)+\left(1-\mu_{A}\left(x_{i}\right)\right) \log \left(1-\mu_{A}\left(x_{i}\right)\right)\right]+(1-\beta) \text {. }
\end{aligned}
$$

It implies

$$
\begin{aligned}
& (1-\beta) \sum_{i=1}^{n} \log \left[D^{n_{i} \mu_{A}\left(x_{i}\right)}\left\{1-\frac{D^{-n_{i}}}{\sum_{j=1}^{n} D^{-n_{j}}}\right\}^{-\left(1-\mu_{A}\left(x_{i}\right)\right)}\right]_{-1} \\
& \leq 2^{(\beta-1)} \sum_{i=1}^{n}\left[\mu_{A}\left(x_{i}\right) \log \mu_{A}\left(x_{i}\right)+\left(1-\mu_{A}\left(x_{i}\right)\right) \log \left(1-\mu_{A}\left(x_{i}\right)\right)\right]+(1-\beta)
\end{aligned}
$$




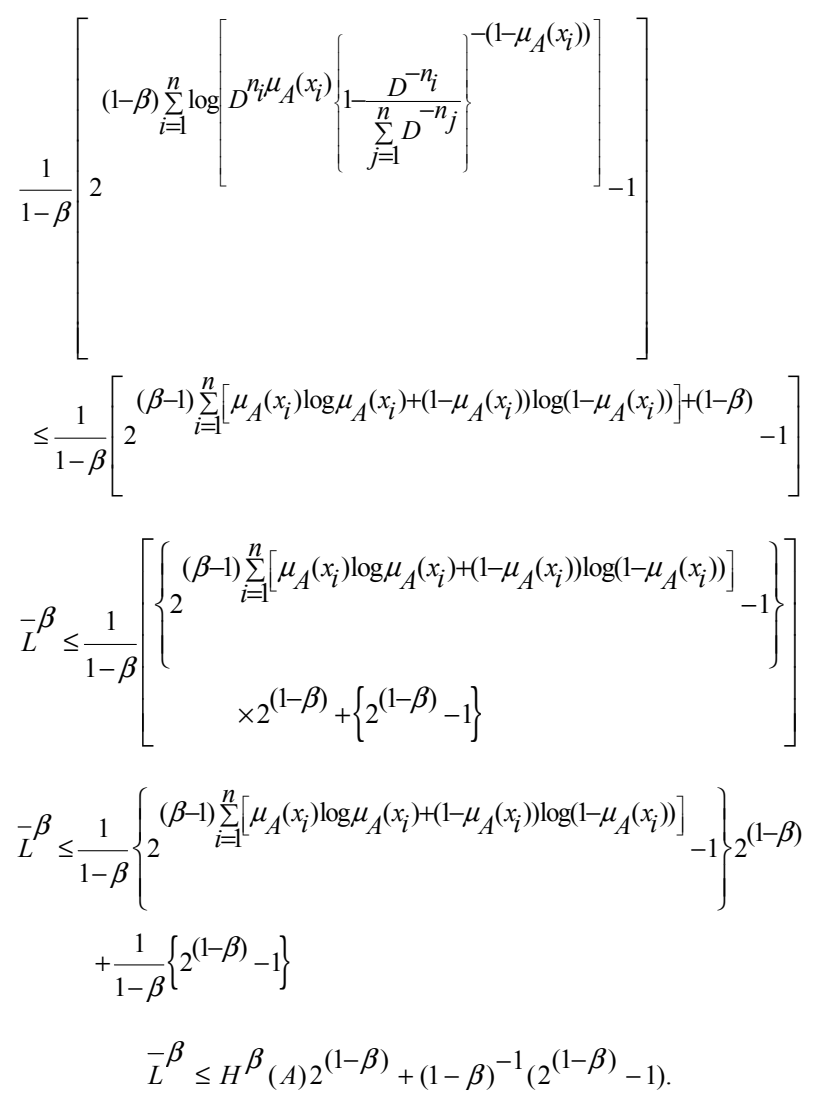

Hence

$$
{ }_{H}^{\beta}(A) \leq \bar{L}^{\beta} \leq H^{\beta}(A) 2^{(1-\beta)}+(1-\beta)^{-1}\left(2^{(1-\beta)}-1\right) .
$$

\section{Particular Case}

When $\beta \rightarrow 1, \bar{L}^{\beta}$ reduces to (6).

$$
\bar{L}=\sum_{i=1}^{n} \log \left[D^{n_{i} \mu_{A}\left(x_{i}\right)}\left\{1-\frac{D^{-n_{i}}}{\sum_{j=1}^{n} D^{-n j}}\right\}^{-\left(1-\mu_{A}\left(x_{i}\right)\right)}\right]
$$

Thus (13) can be called the generalized fuzzy mean code word length of degree $\beta$.

\section{Bounds of Fuzzy Mean Code Word Length of Type $(\alpha, \beta)$}

In this section, we defined fuzzy mean code word length of type $(\alpha, \beta)$.

$$
\begin{aligned}
& { }^{-} \alpha=\frac{1}{2^{1-\alpha}-2^{1-\beta}} \sum_{i=1}^{n}\left[\left\{\mu_{A}^{\alpha}\left(x_{i}\right)+\left(1-\mu_{A}\left(x_{i}\right)\right)^{\alpha}\right\} D^{n_{i}\left(\frac{1-\alpha}{\alpha}\right)}-\left\{\mu_{A}^{\beta}\left(x_{i}\right)+\left(1-\mu_{A}\left(x_{i}\right)\right)^{\beta}\right\} D^{n_{i}\left(\frac{1-\beta}{\beta}\right)}\right] \\
& \text { where } 0<\alpha<1, \beta \geq 1 \text { or } 0<\beta<1, \alpha \geq 1 \text {. }
\end{aligned}
$$

Theorem 2. For all uniquely decipherable codes, noiseless coding theorem states:

$$
H_{\alpha}^{\beta}(A) \leq-\bar{L} \alpha \leq H_{\alpha}^{\beta}+\frac{1}{2^{1-\alpha}-2^{1-\beta}}\left\{D^{\left(\frac{1-\alpha}{\alpha}\right)}-D^{\left(\frac{1-\beta}{\beta}\right)}\right\}
$$

where $H_{\alpha}^{\beta}(A)$ is given by (12).

Proof. By Hölder's inequality, we have

$\sum_{i=1}^{n} x_{i} y_{i} \geq\left(\sum_{i=1}^{n} x_{i}^{p}\right)^{\frac{1}{p}}\left(\sum_{i=1}^{n} y_{i}^{q}\right)^{\frac{1}{q}} ; 0<p<1, q<0$ or $0<q<1, p<0$

and

$\sum_{i=1}^{n} x_{i} y_{i} \leq\left(\sum_{i=1}^{n} x_{i}^{p}\right)^{\frac{1}{p}}\left(\sum_{i=1}^{n} y_{i}^{q}\right)^{\frac{1}{q}} ; \frac{1}{p}+\frac{1}{q}=1, p \geq 1, q>1$ and $x_{i}, y_{i}>0$.

From (19), we have

$$
\sum_{i=1}^{n} x_{i} y_{i} \geq\left(\sum_{i=1}^{n} x_{i}^{p}\right)^{\frac{1}{p}}\left(\sum_{i=1}^{n} y_{i}^{q}\right)^{\frac{1}{q}} ; 0<p<1, q<0 \text { or } 0<q<1, p<0 .
$$

$$
\text { Set } x_{i}=\left[f\left(\mu_{A}\left(x_{i}\right), \mu_{B}\left(x_{i}\right)\right)\right]^{-\frac{1}{t}} D^{-n_{i}}, y_{i}=\left[f\left(\mu_{A}\left(x_{i}\right), \mu_{B}\left(x_{i}\right)\right)\right]^{\frac{1}{t}} \text { and } p=-t, q=\frac{t}{1+t} \text {. }
$$

Then

$$
\sum_{i=1}^{n} D^{-n_{i}} \geq\left[\sum_{i=1}^{n} f\left(\mu_{A}\left(x_{i}\right), \mu_{B}\left(x_{i}\right)\right) D^{n_{i} t}\right]^{\frac{-1}{t}}\left[\sum_{i=1}^{n} f\left(\mu_{A}\left(x_{i}\right), \mu_{B}\left(x_{i}\right)\right)\right]^{\frac{1}{t}}
$$
by Kraft's inequality

$$
\left[\sum_{i=1}^{n} f\left(\mu_{A}\left(x_{i}\right), \mu_{B}\left(x_{i}\right)\right) D^{n_{i} t}\right]^{\frac{-1}{t}}\left[\sum_{i=1}^{n} f\left(\mu_{A}\left(x_{i}\right), \mu_{B}\left(x_{i}\right)\right)\right]^{\frac{1}{t}} \leq \sum_{i=1}^{n} D^{-n_{i}} \leq 1
$$

or

$$
\left[\sum_{i=1}^{n} f\left(\mu_{A}\left(x_{i}\right), \mu_{B}\left(x_{i}\right)\right)\right] \leq\left[\sum_{i=1}^{n} f\left(\mu_{A}\left(x_{i}\right), \mu_{B}\left(x_{i}\right)\right) D^{n_{i} t}\right] .
$$

Subtracting $n$ from both sides, we get

$$
\sum_{i=1}^{n}\left\{f\left(\mu_{A}\left(x_{i}\right), \mu_{B}\left(x_{i}\right)\right)-1\right\} \leq \sum_{i=1}^{n}\left\{f\left(\mu_{A}\left(x_{i}\right), \mu_{B}\left(x_{i}\right)\right) D^{n_{i} t}-1\right\} .
$$

Setting $\alpha=\frac{1}{1+t}, \alpha>0, t=\frac{1-\alpha}{\alpha}$ and

$$
f\left(\mu_{A}\left(x_{i}\right), \mu_{B}\left(x_{i}\right)\right)=\mu_{A}^{\alpha}\left(x_{i}\right)+\left(1-\mu_{A}\left(x_{i}\right)\right)^{\alpha} .
$$

Then 


$$
\begin{aligned}
& \frac{1}{2^{1-\alpha}-2^{1-\beta}} \sum_{i=1}^{n}\left[\mu_{A}^{\alpha}\left(x_{i}\right)+\left(1-\mu_{A}\left(x_{i}\right)\right)^{\alpha}-1\right] \\
& \leq \frac{1}{2^{1-\alpha}-2^{1-\beta}} \sum_{i=1}^{n}\left[\mu_{A}^{\alpha}\left(x_{i}\right)+\left(1-\mu_{A}\left(x_{i}\right)\right)^{\alpha} D^{n_{i}\left(\frac{1-\alpha}{\alpha}\right)}-1\right] \text {. }
\end{aligned}
$$

Changing $\alpha$ to $\beta$, we get

$$
\begin{aligned}
& \frac{1}{2^{1-\beta}-2^{1-\alpha}} \sum_{i=1}^{n}\left[\mu_{A}^{\beta}\left(x_{i}\right)+\left(1-\mu_{A}\left(x_{i}\right)\right)^{\beta}-1\right] \\
& \leq \frac{1}{2^{1-\beta}-2^{1-\alpha}} \sum_{i=1}^{n}\left[\mu_{A}^{\beta}\left(x_{i}\right)+\left(1-\mu_{A}\left(x_{i}\right)\right)^{\beta}{ }^{n_{i}\left(\frac{1-\beta}{\beta}\right)}-1\right] .
\end{aligned}
$$

Adding (21) and (22), we get

$\frac{1}{2^{1-\alpha}-2^{1-\beta}} \sum_{i=1}^{n}\left[\mu_{A}^{\alpha}\left(x_{i}\right)+\left(1-\mu_{A}\left(x_{i}\right)\right)^{\alpha}-\mu_{A}^{\beta}\left(x_{i}\right)+\left(1-\mu_{A}\left(x_{i}\right)\right)^{\beta}\right]$

$\leq \frac{1}{2^{1-\alpha}-2^{1-\beta}} \sum_{i=1}^{n}\left[\left\{\mu_{A}^{\alpha}\left(x_{i}\right)+\left(1-\mu_{A}\left(x_{i}\right)\right)^{\alpha}\right\} D^{n_{i}\left(\frac{1-\alpha}{\alpha}\right)}-\left\{\mu_{A}^{\beta}\left(x_{i}\right)+\left(1-\mu_{A}\left(x_{i}\right)\right)^{\beta}\right\} D^{n_{i}\left(\frac{1-\beta}{\beta}\right)}\right]$

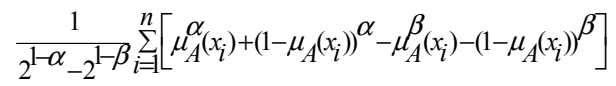

$\leq \frac{1}{2^{1-\alpha}-2^{1-\beta}} \sum_{i=1}^{n}\left[\left\{\mu_{A}^{\alpha}\left(x_{i}\right)+\left(1-\mu_{A}\left(x_{i}\right)\right)^{\alpha}\right\}^{n_{i}\left(\frac{1-\alpha}{\alpha}\right)}-\left\{\mu_{A}^{\beta}\left(x_{i}\right)+\left(1-\mu_{A}\left(x_{i}\right)\right)^{\beta}\right\} D^{n_{i}\left(\frac{1-\beta}{\beta}\right)}\right]$

Hence $H_{\alpha}^{\beta}(A) \leq \bar{L} \alpha$.

Now, we have to prove

$$
\begin{gathered}
\bar{L}_{\alpha}^{\beta} \leq H_{\alpha}^{\beta}+\frac{1}{2^{1-\alpha}-2^{1-\beta}}\left\{D^{\left(\frac{1-\alpha}{\alpha}\right)}-D^{\left(\frac{1-\beta}{\beta}\right)}\right\} \\
\bar{L} \alpha \leq H_{\alpha}(A)+\frac{1}{1-\alpha}\left\{D^{\left(\frac{1-\alpha}{\alpha}\right)}-1\right\} \\
\frac{1}{1-\alpha} \sum_{i=1}^{n}\left[\left\{\mu_{A}^{\alpha}\left(x_{i}\right)+\left(1-\mu_{A}\left(x_{i}\right)\right)^{\alpha}\right\} D^{n_{i}\left(\frac{1-\alpha}{\alpha}\right)}-1\right] \\
\leq \frac{1}{1-\alpha} \sum_{i=1}^{n}\left[\mu_{A}^{\alpha}\left(x_{i}\right)+\left(1-\mu_{A}\left(x_{i}\right)\right)^{\alpha}-1\right]+\frac{1}{1-\alpha}\left\{D^{\left(\frac{1-\alpha}{\alpha}\right)}-1\right\} .
\end{gathered}
$$

It implies

$$
\begin{aligned}
& \sum_{i=1}^{n}\left[\left\{\mu_{A}^{\alpha}\left(x_{i}\right)+\left(1-\mu_{A}\left(x_{i}\right)\right)^{\alpha}\right\}_{D} D_{i}\left(\frac{1-\alpha}{\alpha}\right)-1\right] \\
& \leq \sum_{i=1}^{n}\left[\mu_{A}^{\alpha}\left(x_{i}\right)+\left(1-\mu_{A}\left(x_{i}\right)\right)^{\alpha}-1\right]+\left\{D^{\left(\frac{1-\alpha}{\alpha}\right)}-1\right\} .
\end{aligned}
$$

Similarly, we can prove that

$$
\bar{L} \beta \leq H_{\beta}(A)+\frac{1}{1-\beta}\left\{D^{\left(\frac{1-\beta}{\beta}\right)}-1\right\} \text {. }
$$

It implies

$$
\begin{aligned}
\sum_{i=1}^{n} & {\left[\left\{\mu_{A}^{\beta}\left(x_{i}\right)+\left(1-\mu_{A}\left(x_{i}\right)\right)^{\beta}\right\} D^{n_{i}\left(\frac{1-\beta}{\beta}\right)}-1\right] } \\
& \leq \sum_{i=1}^{n}\left[\mu_{A}^{\beta}\left(x_{i}\right)+\left(1-\mu_{A}\left(x_{i}\right)\right)^{\beta}-1\right]+\left\{D^{\left(\frac{1-\beta}{\beta}\right)}-1\right\} .
\end{aligned}
$$

From (25) and (27), we have

$$
\begin{aligned}
& \frac{1}{2^{1-\alpha}-2^{1-\beta}} \sum_{i=1}^{n}\left[\left\{\mu_{A}^{\alpha}\left(x_{i}\right)+\left(1-\mu_{A}\left(x_{i}\right)\right)^{\alpha}\right\}_{D}{ }^{n_{i}\left(\frac{1-\alpha}{\alpha}\right)}-1\right] \\
& \leq \frac{1}{2^{1-\alpha}-2^{1-\beta}}\left[\sum_{i=1}^{n}\left[\mu_{A}^{\alpha}\left(x_{i}\right)+\left(1-\mu_{A}\left(x_{i}\right)\right)^{\alpha}-1\right]+\left\{D^{\left(\frac{1-\alpha}{\alpha}\right)}-1\right\}\right. \text {. }
\end{aligned}
$$

and

$$
\begin{aligned}
& \frac{1}{2^{1-\beta}-2^{1-\alpha}} \sum_{i=1}^{n}\left[\left\{\mu_{A}^{\beta}\left(x_{i}\right)+\left(1-\mu_{A}\left(x_{i}\right)\right)^{\beta}\right\} D^{n_{i}\left(\frac{1-\beta}{\beta}\right)}-1\right] \\
& \leq \frac{1}{2^{1-\beta}-2^{1-\alpha}}\left[\sum_{i=1}^{n}\left[\mu_{A}^{\beta}\left(x_{i}\right)+\left(1-\mu_{A}\left(x_{i}\right)\right)^{\beta}-1\right]+\left\{D^{\left(\frac{1-\beta}{\beta}\right)}-1\right\} .\right.
\end{aligned}
$$

Adding (28) and (29), we have

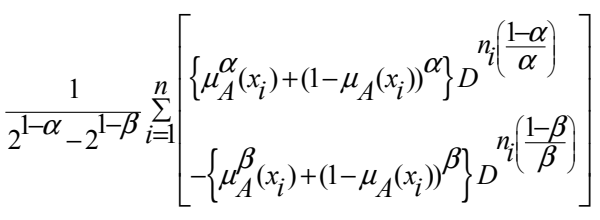

$$
\begin{aligned}
& \leq \frac{1}{2^{1-\alpha}-2^{1-\beta}}\left[\begin{array}{c}
\sum_{i=1}^{n}\left[\mu_{A}^{\alpha}\left(x_{i}\right)+\left(1-\mu_{A}\left(x_{i}\right)\right)^{\alpha}-\mu_{A}^{\beta}\left(x_{i}\right)+\left(1-\mu_{A}\left(x_{i}\right)\right)^{\beta}\right] \\
+\left\{D_{-D}^{\left(\frac{1-\alpha}{\alpha}\right)}{ }^{\left(\frac{1-\beta}{\beta}\right)}\right\}
\end{array}\right]
\end{aligned}
$$

Hence

$$
H_{\alpha}^{\beta}(A) \leq \bar{L}_{\alpha}^{\beta} \leq H_{\alpha}^{\beta}+\frac{1}{2^{1-\alpha}-2^{1-\beta}}\left\{D^{\left(\frac{1-\alpha}{\alpha}\right)}-D^{\left(\frac{1-\beta}{\beta}\right)}\right\} .
$$

Particular Cases

1. When $\alpha=1$ and $\beta \rightarrow 1, \bar{L} \alpha$ reduces to

$\bar{L}=-\frac{1}{\log 2} \sum_{i=1}^{n}\left[\mu_{A}\left(x_{i}\right) \log \mu_{A}\left(x_{i}\right)+\left(1-\mu_{A}\left(x_{i}\right)\right) \log \left(1-\mu_{A}\left(x_{i}\right)\right)-n_{i} \log D\right]$. 
2. When $\alpha \rightarrow 1$ and $\beta=1,-\bar{L} \alpha$ reduces to

$\bar{L}=-\frac{1}{\log 2} \sum_{i=1}^{n}\left[\mu_{A}\left(x_{i}\right) \log \mu_{A}\left(x_{i}\right)+\left(1-\mu_{A}\left(x_{i}\right)\right) \log \left(1-\mu_{A}\left(x_{i}\right)\right)-n_{i} \log D\right]$

Thus (17) can be called fuzzy mean code word length of type $(\alpha, \beta)$.

\section{Monotonic Behavior of Fuzzy Mean Code Word Lengths}

In this section, we study analytically the monotonic behavior of fuzzy mean code word length $\bar{L}^{\beta}$.

From equation (13), we have

$$
\left.\bar{L}^{\beta}=\frac{1}{1-\beta}\left[2^{(1-\beta) \sum_{i=1}^{n} \log \left(D^{n_{i} \mu_{A}\left(x_{i}\right)}\left(1-\frac{D^{-n_{i}}}{\sum_{j=1}^{n} D^{-n_{j}}}\right)^{-\left(1-\mu_{A}\left(x_{i}\right)\right)}\right\}}\right\}_{-1}\right],
$$

Equation (30) can be rewritten as

$$
{ }^{-} \beta=\frac{1}{1-\beta}\left[2^{(1-\beta) N}-1\right], \beta \neq 1, \beta>0,
$$

where

$$
N=\sum_{i=1}^{n}\left[\log \left\{D^{n_{i} \mu_{A}\left(x_{i}\right)}\left(1-\frac{D^{-n_{i}}}{\sum_{j=1}^{n} D^{-n_{j}}}\right)^{-\left(1-\mu_{A}\left(x_{i}\right)\right)}\right\} \geq 0 .\right.
$$

Differentiating (31) with respect to $\beta$, we have

$$
\begin{gathered}
\frac{d \bar{L}^{\beta}}{d \beta}=\frac{1}{(1-\beta)^{2}}\left\{2^{(1-\beta) N}-1\right\}+\frac{N \log 2\left\{2^{(1-\beta) N}\right\}}{(1-\beta)} . \\
\frac{d \bar{L} \beta}{d \beta}=\frac{1}{(1-\beta)^{2}}\left[2^{(1-\beta) N}\left\{1+\log 2^{(1-\beta) N}\right\}-1\right] .
\end{gathered}
$$

Here two cases arise:

Case 1: When $\beta<1$, we have $\frac{d \bar{L}}{d \beta}<0$,

which shows that $\bar{L}^{\beta}$ is a monotonically decreasing function of $\beta$ and $\beta<1$.

The above result is verified by plotting the graphs on MATLAB for different values of $\beta$ and $\beta<1$.

From below figure we can generalize that the value of

$\bar{L}^{\beta}$ decreases with respect to $\beta$.

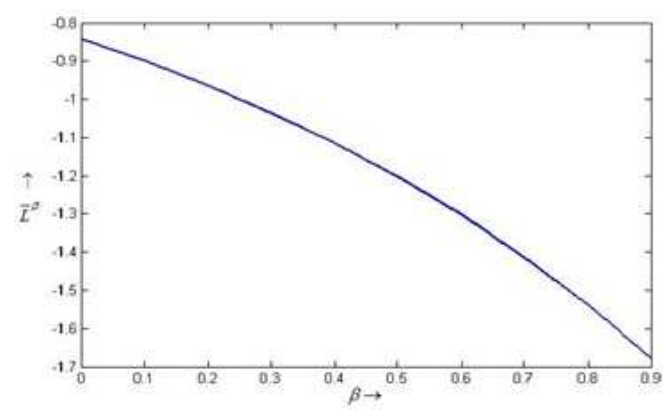

Figure 1. Relation between $\bar{L}$ and $\beta(<0)$.

Case 2: When $\beta>1$, we have $\frac{d \bar{L}^{\beta}}{d \beta}<0$,

which shows that $\bar{L}^{\beta}$ is a monotonically decreasing function of $\beta$.

The above result is verified by plotting the graphs on MATLAB for different values of $\beta$ and $\beta>1$.

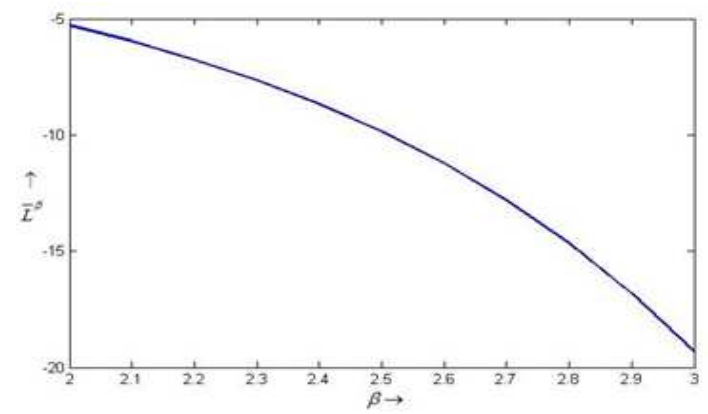

Figure 2. Relation between $-\beta$ and $\beta(>0)$.

From above figure we can generalize that the value of $\bar{L}^{\beta}$ monotonically decreases with respect to $\beta$ and $\beta>1$. We have also studied the monotonic behavior of fuzzy mean code word length $\bar{L} \alpha$.

From equation (17), we have

$$
\bar{L}_{\alpha}^{\beta}=\frac{1}{2^{1-\alpha}-2^{1-\beta}} \sum_{i=1}^{n}\left[\begin{array}{c}
\left\{\mu_{A}^{\alpha}\left(x_{i}\right)+\left(1-\mu_{A}\left(x_{i}\right)\right)^{\alpha}\right\}_{D}{ }^{n_{i}\left(\frac{1-\alpha}{\alpha}\right)} \\
-\left\{\mu_{A}^{\beta}\left(x_{i}\right)+\left(1-\mu_{A}\left(x_{i}\right)\right)^{\beta}\right\}_{D}{ }^{n_{i}\left(\frac{1-\beta}{\beta}\right)}
\end{array}\right] .
$$

Equation (32) can be rewritten as

$$
\bar{L} \beta=\frac{1}{2^{1-\alpha}-2^{1-\beta}}[(1-\alpha) \bar{L} \alpha-(1-\beta) \bar{L} \beta]
$$

where 
$\bar{L} \alpha=\frac{1}{1-\alpha} \sum_{i=1}^{n}\left[\left\{\mu_{A}^{\alpha}\left(x_{i}\right)+\left(1-\mu_{A}\left(x_{i}\right)\right)^{\alpha}\right\} D^{n_{i}\left(\frac{1}{\alpha}-1\right)}-1\right]$

and

$\bar{L} \beta=\frac{1}{1-\beta} \sum_{i=1}^{n}\left[\left\{\mu_{A}^{\beta}\left(x_{i}\right)+\left(1-\mu_{A}\left(x_{i}\right)\right)^{\beta}\right\} D^{n_{i}\left(\frac{1}{\beta}-1\right)}-1\right]$.

Differentiating (33) with respect to $\alpha$, we have

$$
\begin{array}{r}
\frac{\partial \bar{L} \alpha}{\partial \alpha}=\frac{2^{1-\alpha} \log 2}{\left(2^{1-\alpha}-2^{1-\beta}\right)^{2}}[(1-\alpha) \bar{L} \alpha-(1-\beta) \bar{L} \beta] \\
+\frac{1}{2^{1-\alpha}-2^{1-\beta}}\left[(1-\alpha) \frac{d \bar{L} \alpha}{d \alpha}-\bar{L} \alpha\right]
\end{array}
$$

$\frac{\partial \bar{L} \beta}{\partial \alpha}=\frac{2^{1-\alpha} \bar{L} \alpha\{(1-\alpha) \log 2-1\}+\bar{L} \alpha 2^{1-\beta}-2^{1-\alpha}(1-\beta) \bar{L} \beta \log 2}{\left(2^{1-\alpha}-2^{1-\beta}\right)^{2}}$

$$
+\frac{(1-\alpha)}{2^{1-\alpha}-2^{1-\beta}} \frac{d \bar{L} \alpha}{d \alpha}
$$

Here two cases arise:

Case 1: When $0<\alpha<1, \beta>1, \bar{L} \alpha>\bar{L} \beta$, we get

$$
\frac{\partial \bar{L} \alpha}{\partial \alpha}>0
$$

which shows that $\bar{L}_{\alpha}^{\beta}$ is a monotonically increasing function of $\alpha$. The above result is verified by plotting the graphs on MATLAB for different values of $\alpha(\alpha<1)$ and fixed value of $\beta$.

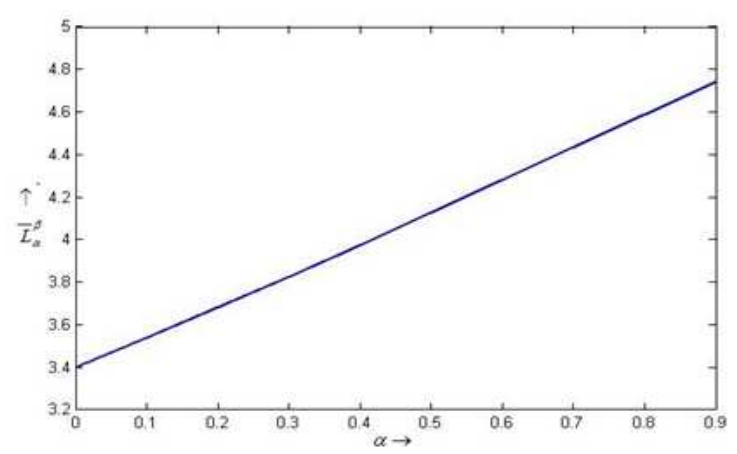

Figure 3. Relation between $\bar{L} \alpha$ and $\alpha(<0)$.

Case 2: When $\alpha>1,0<\beta<1, \bar{L} \alpha<\bar{L} \beta$, we have

$$
\frac{\partial \bar{L} \alpha}{\partial \alpha}>0
$$

which shows that $\bar{L} \boldsymbol{\alpha}$ is a monotonically decreasing function of $\alpha$. The above result is verified by plotting the graphs on MATLAB for different values of $\alpha(\alpha>1)$ and fixed values of $\beta$.

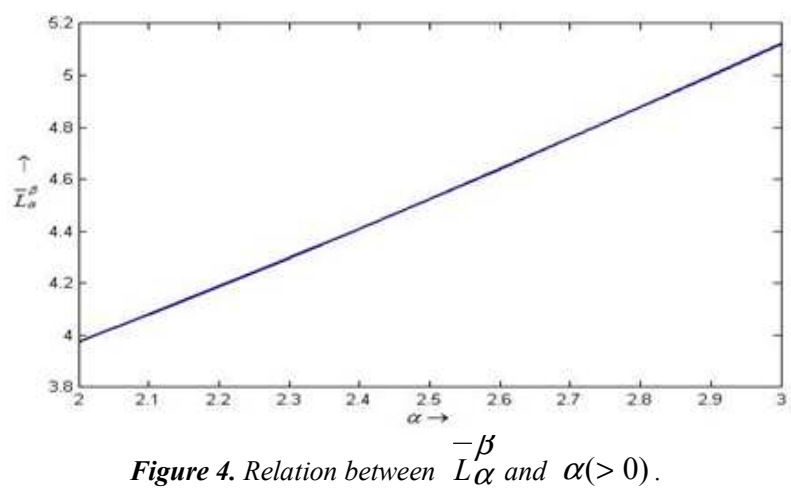

Again differentiating (33) with respect to $\beta$, we have

$$
\begin{gathered}
\frac{\partial \bar{L} \alpha}{\partial \beta}=\frac{-2^{1-\beta} \log 2}{\left(2^{1-\alpha}-2^{1-\beta}\right)^{2}}[(1-\alpha) \bar{L} \alpha-(1-\beta) \bar{L} \beta] \\
-\frac{1}{2^{1-\alpha}-2^{1-\beta}}\left[(1-\beta) \frac{d \bar{L} \beta}{d \alpha}-\bar{L} \beta\right] . \\
\frac{\partial \bar{L} \alpha}{\partial \beta}=\frac{2^{1-\beta} \frac{{ }^{-} \beta\{(1-\beta) \log 2-1\}+\bar{L} \beta 2^{1-\alpha}-2^{1-\beta}(1-\alpha) \bar{L} \alpha \log 2}{\left(2^{1-\alpha}-2^{1-\beta}\right)^{2}}}{-\frac{(1-\beta)}{2^{1-\alpha}-2^{1-\beta} \frac{d \bar{L} \beta}{d \beta} .}}
\end{gathered}
$$

Here two cases arise:

Case 1: When $0<\alpha<1, \beta>1, \bar{L} \alpha>\bar{L} \beta$, we get

$$
\frac{\partial \bar{L} \alpha}{\partial \beta}>0
$$

which shows that $\bar{L} \alpha$ is a monotonically increasing function of $\beta$.

Case 2: When $\alpha>1,0<\beta<1, \bar{L} \alpha<\bar{L} \beta$, we have

$$
\frac{\partial \bar{L} \alpha}{\partial \beta}>0
$$

which shows that $\bar{L} \alpha$ is a monotonically increasing function of $\beta$. 


\section{References}

[1] A. De Luca and S. Termini, "A definition of non probabilistic entropy in setting of fuzzy set theory", Inform. Contr., 20 (972), pp. 301-312.

[2] A. Renyi, "On measures of entropy and information", Proceedings $4^{\text {th }}$ Berkeley Symposium on Mathematical Statistics and Probability, 1 (1961), 547-561.

[3] B. D. Sharma and D. P. Mittal, "New non- additive measures of entropy for discrete probability distributions", J. Math. Sci (Calcutta), 10 (1975), 28-40.

[4] B. D. Sharma and I. J. Taneja, "Three generalized additive measures of entropy”, Elec. Inform. Kybern., 13 (1977), pp. 419-433.

[5] C. E. Shannon, "A mathematical theory of communication", Bell Syst. Tech. J., 27 (1948), pp. 379-423 \& 623-659.

[6] D. Bhandari, N. R. Pal and D.D. Majumder, "Fuzzy divergence, probability measure of fuzzy events and image thresholding", Pattern Recognition Letters, 1 (1992), 857-867.

[7] D. S. Hooda and Divya Jain, "Sub additive measures of fuzzy information", Journal of Reliability and Statistical Studies, 02 (2009), pp. 39-52.

[8] D. S. Hooda, "On generalized measures of fuzzy entropy", Mathematica Slovaka, 54 (2004), pp. 315-325.

[9] J. N. Kapur, "Measures of fuzzy information", Mathematical Science Trust Society, New Delhi (1997).

[10] L. A. Zadeh, "Fuzzy sets", Inform. Contr., 8 (1965), pp. 338-353.

[11] L. G. Kraft, "A device for quantizing grouping and coding amplitude modulated pulses", M. S. Thesis, Electrical Engineering Department, MIT (1949).

[12] O. Prakash and P. K. Sharma, "Noiseless coding theorems corresponding to fuzzy entropies", Southeast Asian Bulletin of Mathematics, 27 (2004), 1073-1080. 